\title{
Morphologic changes of diving-related barotrauma lung injury in the setting of Birt-Hogg-Dubé
}

\author{
Maria J. Merino*1, Marston Linehan ${ }^{2}$, Sara Gil ${ }^{1}$ \\ ${ }^{1}$ Translational Surgical Pathology, Laboratory of Pathology, National Cancer Institute, National Institutes of Health, United \\ States \\ ${ }^{2}$ Urologic Oncology Branch, Center for Cancer Research, National Cancer Institute, National Institutes of Health, United States
}

Received: May 30, 2017

DOI: $10.5430 /$ css.v3n $4 p 25$
Accepted: August 24, 2017

Online Published: November 20, 2017

\begin{abstract}
Manifestations of barotrauma-associated lung injury with scuba diving are rarely reported, aside from anecdotic historical accounts. The pathology of the lung changes has not been adequately characterized. We report the histologic findings of diving-related chronic lung injury in a patient with Birt-Hogg-Dube (BHD). The histologic findings included bullae, chronic pleuritis, edema, vascular and interstitial thickening. In addition, there were multiple necrotizing granulomas containing fungal organisms. BHD is a hereditary condition characterized by skin, lung, and renal lesions. This is to our knowledge the first report of the histologic findings of diving-related pulmonary barotrauma (PBT) injury, albeit in the setting of BHD.
\end{abstract}

Key Words: Barotrauma, Pulmonary, Spontaneous pneumothorax, Birt-Hogg-Dubé, Histopathology

\section{INTRODUCTION}

Despite the increasing popularity of diving, pulmonary barotrauma (PBT) with or without decompression sickness remains a relatively infrequent medical complication. Education and technological advancements have made this condition rare, and the clinical history and presentation typically does not warrant pathology evaluation of tissue to ascertain a diagnosis. Patients present with symptoms characteristic of pneumothorax, pneumomediastinum, or signs suggestive of arterial emboli. This is usually corroborated by conventional imaging in the acute or sub-acute period, showing subpleural bullae and blebs, interstitial emphysema, lung cysts and ectopic air. ${ }^{[1]}$ However, chronic PBT changes in the lung may not be distinguishable from other lesions by traditional imaging. Needle core biopsy of the lung in general supply an chance to set up a relatively non-invasive pathological diagnosis to backing appropriate clinical management. The number of case reports describing pathological findings of PBT of the lung is limited by its unusual and clinical diagnosis. In the incoming report we depict the morphologic changes of PBT chronic lung injury, in the setting of BirtHogg-Dube (BHD).

\section{CASE REPORT}

An apparently wholesome 32-year-old man member of a family with known BHD came for an initial evaluation and screening at the National Institutes of Health, in the BHD protocol. On physical examination, he had numerous fibrofolliculomas in the skin consistent with BHD. He gave a history of pneumothoraces; a pneumothorax at the age of 15 after some trauma, and in June 2008 experienced a complete right pneumothorax with air embolism during ascent of

\footnotetext{
*Correspondence: Maria J. Merino; Email: mjmerino@mail.nih.gov; Address: Laboratory of Pathology, National Cancer Institute, National Institutes of Health, 10 Center Drive, Building 10, Office 3S235C, Bethesda, United States.
} 
compressed air diving. Otherwise he was in good medical health. An abdominal computed tomograph (CT) did not show evidence of any renal lesions. However, CT imaging of the lungs showed pulmonary parenchyma with numerous emphysematous bullae, blebs and pulmonary cysts. In addition, a well-circumscribed lesion was noted contiguous, but external to the bullae with hyperattenuation Proposing a firm mass (see Figure 1). The differential diagnosis included infection, abscess, pulmonary cyst with solid component, hamartoma and neoplasm. The mass estimated to measure $3 \mathrm{~cm} \times 2 \mathrm{~cm}$. Given the patient's diagnosis of BHD, a neoplastic lesion could not be excluded and sampling was warranted. A needle-core biopsy showed only necrotic tissue. The possibility of necrotic tumor or an infectious process was considered and it was decided to excise to further elucidate the lesion. The patient had an uneventful recuperation and follow up.

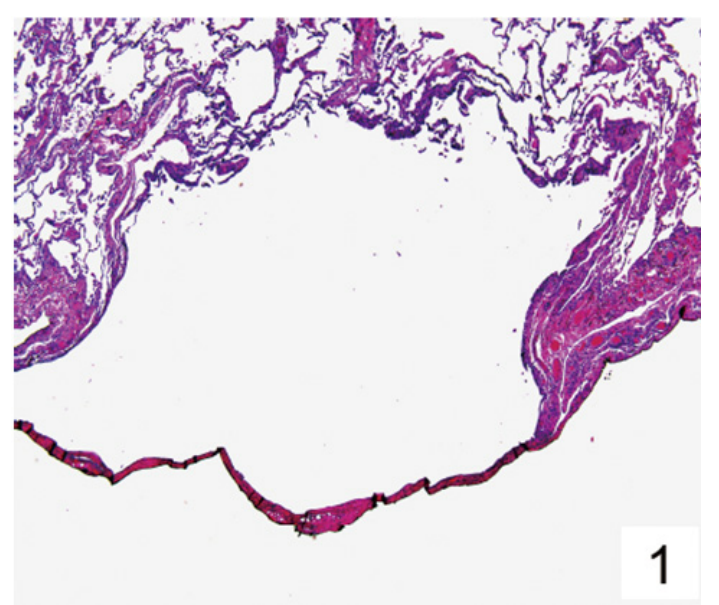

Figure 1. Bullae with hyperattenuation, hematoxylin and eosin stain (H\&E)

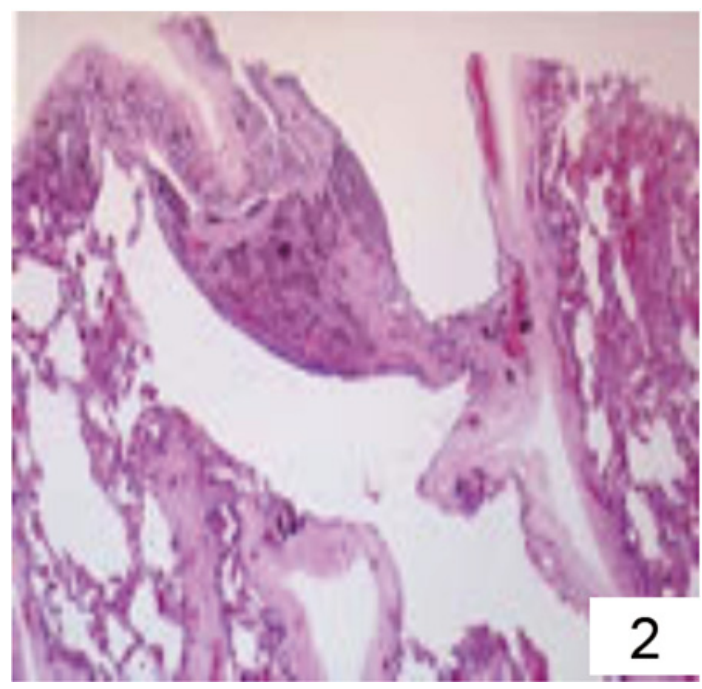

Figure 2. Pulmonary edema (H\&E)
The medical team warned the patient of the dangers of continuing to practice diving, especially due to pulmonary complications such as Pneumothorax, and gave information to him and his family about increased risk of renal neoplasms.

\section{Macroscopic and histologic detection}

The specimen consisted of a left lower lobe wedge resection measuring $15 \mathrm{~cm} \times 4 \mathrm{~cm} \times 3.5 \mathrm{~cm}$. The pleural surface was opaque and showed multiple blebs. Subsequent bivalving revealed a $2.5-\mathrm{cm}$ white firm circumscribed nodule. The lesion was focally necrotic and friable. The surrounding pulmonary parenchyma showed numerous dilated airways and bullae. Histologically, the nodule showed extensive necrosis with chronic inflammation in the periphery. An abnormal vessel was discerned in the center of the lesion. Diffuse pleural bullae with associated chronic pleuritis were present. The lung parenchyma showed scattered dilated cystic spaces consistent with emphysematous changes. Pulmonary edema was focally present (see Figure 2). The vessels showed prominent thickening of their wall and some showed partial occlusion of their lumen (see Figure $3 a$ and $3 b$ ). In the remaining parenchyma, small non-necrotizing granulomata were identified, containing fungal organisms consistent with Histoplasma capsulatum (see Figure 4).

\section{DisCUSSION}

In 1942, Jacques-Yves Cousteau and Emile Gagnan invented a device coined the "Aqua Lung" the predecessor to modern scuba apparati allowing divers to plunge to unforeseen depths. In the advent of technology, PBT became an infrequent but important complication, due to tissue injury resulting from expanding gas during rapid ascent, precluding sufficient or appropriate exhalation. According to Boyle's Law, the volume of a given mass of gas is inversely proportional to the absolute pressure, whereas during ascent as the ambient pressure decreases, gas inside the lungs expands. The expansion of compressed air in the lungs can lead to pulmonary tissue damage, pneumothorax, pneumomediastinum, and most seriously arterial air emboli. Persons with history of trauma, previous pneumothorax, ${ }^{[2]}$ lung cysts, ${ }^{[3]}$ emphysema ${ }^{[4]}$ or genetic conditions like cystic fibrosis, Ehlers Danlos, and Marfans ${ }^{[5]}$ are predisposed to develop sequelae, and precluded from diving. Our patient was diagnosed, secondary to family history and clinical constellation with Birt-HoggDubé, a genodermatosis characterized by fibrofolliculomas, renal tumors, and spontaneous pneumothorax, a condition also obviating fitness to dive. ${ }^{[6]} \mathrm{CT}$ imaging has shown that pulmonary injury from PBT tends to resolve quickly, however persistence may remain in patients with preexisting lung injury, ${ }^{[7]}$ akin to our case. Despite utilization of radiographic techniques, these findings are not diagnostic in 
patients with delayed presentation, and may be suggestive of a more aggressive lesion. Published case reports of the histologic findings of PBT injury identified rarely describe or illustrate the morphologic changes associated with PBT lung injury in compressed air diving. ${ }^{[8-10]}$

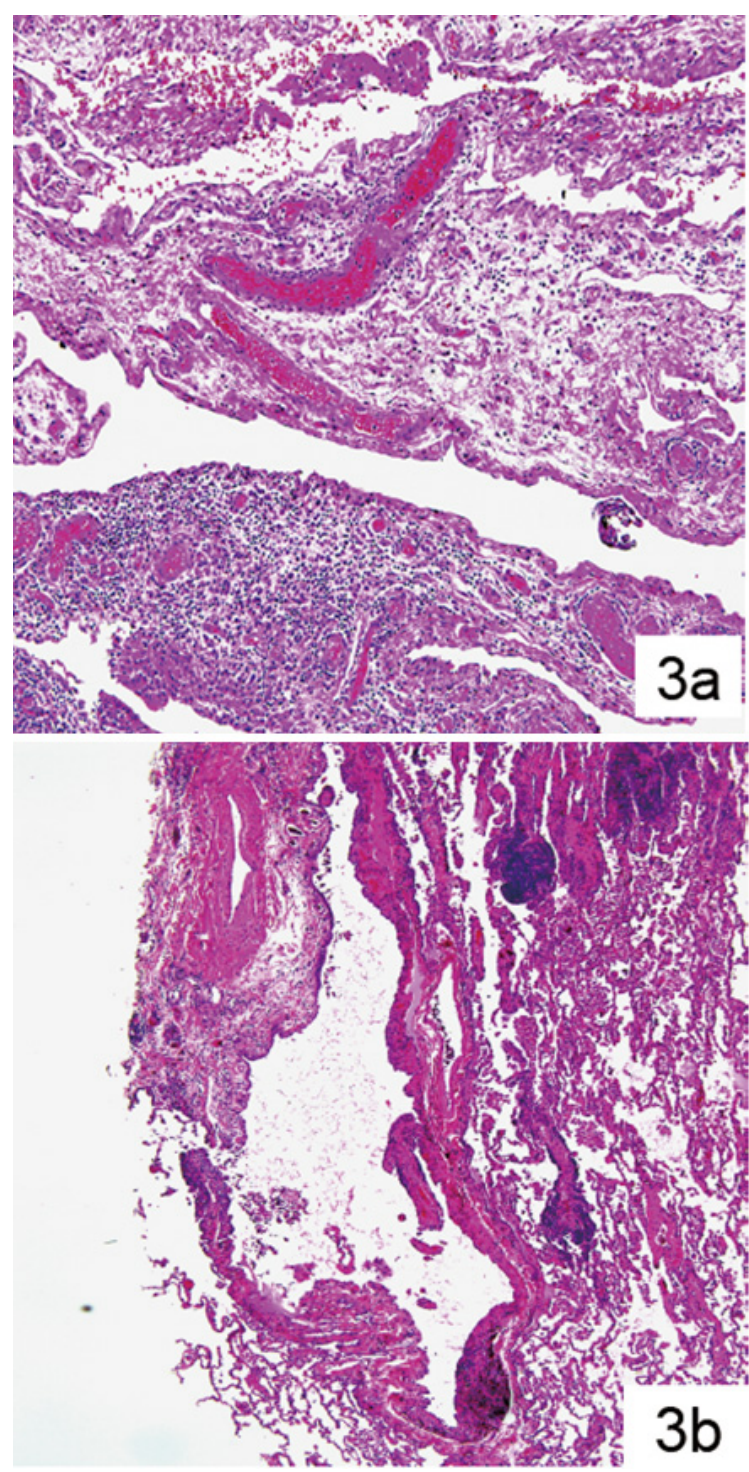

Figure 3. Vessels with prominent thickening of their wall and some with partial occlusion of their lumen (H\&E, 3a and $3 b$ )

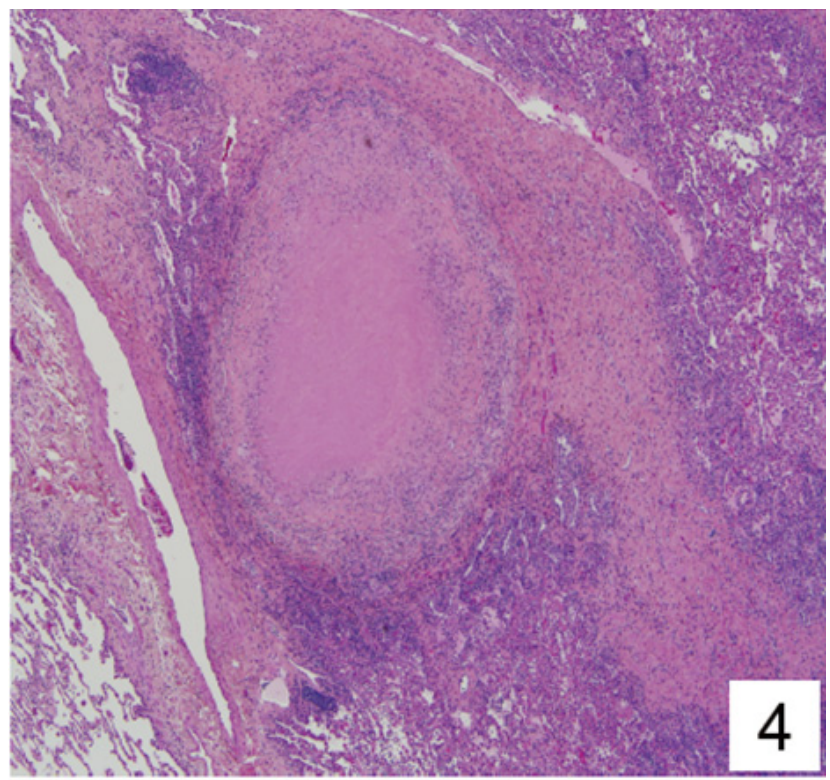

Figure 4. Small non-necrotizing granulomata with fungal organisms consistent with Histoplasma capsulatum (H\&E)

In our case, histologic findings revealed bullae, chronic pleuritis, focal edema, vascular and interstitial thickening. The presence of bullae, could be attributed to either his longstanding history of diving or his hereditary condition. The longstanding history of diving and early symptomatology suggested barotrauma as the most likely cause of the pneumothorax. This case illustrates the limitations sometimes encountered in patients which complex medical histories, allowing an exceptionally rare case of compressed air diving associated PBT injury in the setting of BHD to be elucidated by histological examination of the resected wedge. The histopathologic features of PBT injury in compressed air diving such as air emboli and vascular damage are distinct. The presence of necrotic granulomatous inflammation and fungi was most likely an unrelated event.

\section{CONFLicts OF InTEREST Disclosure}

The authors declare they have no conflicts of interest.

\section{REFERENCES}

[1] Reuter M, Tetzlaff K, Warninghoff V, et al. Computed tomography of the chest in diving-related pulmonary barotrauma. British Journal of Radiology. 1997; 70(833): 440-5. PMid: 9227223. https://doi.org/10.1259/bjr.70.833.9227223

[2] Sadikot RT, Greene T, Meadows K, et al. Recurrence of primary spontaneous pneumothorax. Thorax. 1997; 52: 805-809. PMid: 9371212.

\section{https://doi.org/10.1136/thx.52.9.805}

[3] Simpson G. Primary lung bullae and scuba diving. SPUMS J. 1998; 28: $10-12$.

[4] Mellem H, Emhjellen S, Horgen O. Pulmonary barotrauma and arterial gas embolism caused by an emphysematous bulla in a SCUBA diver. Aviat Space Environ Med. 1990; 61: 559-562. PMid: 2369396. 
[5] Godden D, Currie G, Denison D, et al. British Thoracic Society guidelines on respiratory aspects of fitness for diving. Thorax. 2003; 58: 3-13. https://doi.org/10.1136/thorax.58.1.3

[6] Schmidt LS, Warren MB, Nickerson ML, et al. Birt-Hogg-Dubé syndrome, a genodermatosis associated with spontaneous pneumothorax and kidney neoplasia, maps to chromosome 17p11.2. Am J Hum Genet. 2001; 69: 876-882. PMid: 11533913. https : //doi.org/10.1086/323744

[7] Tetzlaff K, Reuter M, Leplow B, et al. Risk Factors for Pulmonary Barotrauma in Divers Chest. 1997; 112: 654-659. PMid: 9315797.
[8] Chambers HM, van Velzen D. Ventilator-related pathology in the extremely immature lung. Pathology. 1989; 21(2): 79-83. https : //doi.org/10.3109/00313028909059539

[9] Rouby JJ, Lherm T, Martin de Lassale E, et al. Histologic aspects of pulmonary barotrauma in critically ill patients with acute respiratory failure. Intensive Care Med. 1993; 19(7): 383-389. PMid: 8270717. https://doi.org/10.1007/BF01724877

[10] Tsokos M, Paulsen F, Petri S, et al. Histologic, immunohistochemical, and ultrastructural findings in human blast lung injury. Am J Respir Crit Care Med. 2003; 168(5): 549-555. PMid: 12842857. https://doi.org/10.1164/rccm.200304-5280C 